\title{
Call for Special Issue Papers: Tissue Engineering: Part A - Special Issue on Hydrogels and Injectable Systems
}

\section{Deadline for Manuscript Submission: October 20, 2020}

\author{
Guest Editor: Prof. Liumin He; Sun Yat-Sen University, China.
}

\section{General Information}

In native tissues, cells reside within a largely aqueous environment. In this context, hydrogels, which are 3-dimensional (3D) polymeric networks with the ability to hold large quantities of water, have been suggested to be promising in tissue engineering materials by resembling the key physiological characteristics of natural extracellular matrices. Hydrogels have also shown to be outstanding materials to provide structural support for regenerating tissues, particularly hydrogels with injectable and in situ gelling features as they can completely fill the cavity in a noninvasive manner, regardless of the size and shape.

In recent years, various response properties have been introduced to smart hydrogels, such as thermo-response, $\mathrm{pH}$-response, glucose-response, ionrecognition, molecular-recognition. Meanwhile, novel hydrogels have been developed, which have immunoregulatory effects, self-healing properties, controlled-release of drugs, and the ability to specifically induce cell growth and tissue regeneration. Therefore, hydrogels have a wider application in tissue engineering than ever before.

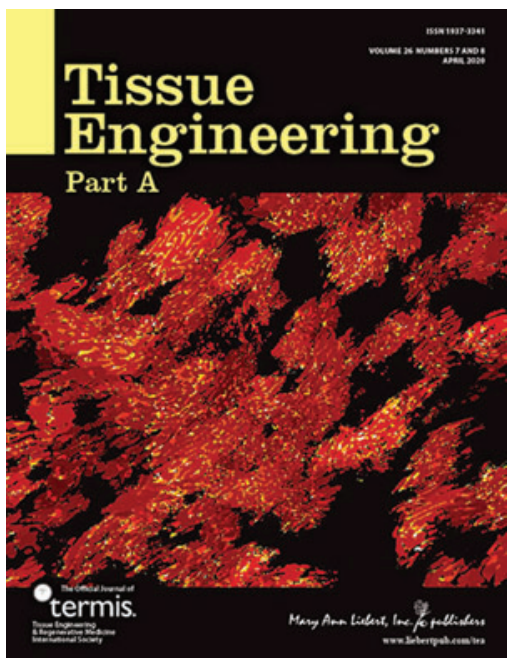

This dedicated special issue on "Hydrogels and Injectable Systems" is dedicated to the development of novel hydrogels for applications in tissue engineering. The influential mechanism of hydrogel properties will be elucidated.

Topics of interest for this special issue include but are not limited to:

- Synthesis of novel hydrogel materials and characterization

- The combination of hydrogels with new processing technologies

- The influences of hydrogel properties on cell biological functions in vitro

- The applications of hydrogels for promoting tissue regeneration in vivo

- Evaluation of clinical efficacy and safety of hydrogels

- Mechanistic insight and modelling

We also encourage submission of papers that have been published in conference proceedings, with the requirement that the authors have made significant extensions as compared to the already published version of the study. For more information, visit the Instructions for Authors at www.liebertpub.com/ten 
Manuscript Submission Deadline: October 20, 2020

Questions or pre-submission queries? Please contact Guest Editor Professor Liumin He at helm9@mail.sysu.edu.cn

Visit the Instructions for Authors:

www.liebertpub.com/ten

\section{Submit your paper for peer review online:}

https://mc.manuscriptcentral.com/ten 